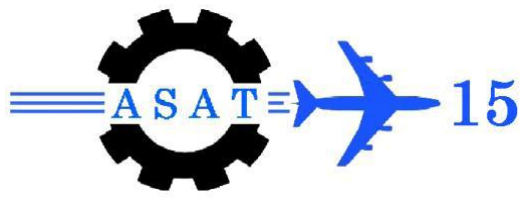

\title{
Dynamics of a Flexible Beam Moving in Earth Orbit
}

\author{
Ahmed Badawy
}

\begin{abstract}
Elastic properties of manoeuvring object orbiting earth is discussed herein. Deflections in an elastic beam produced as a result of its motion and external forces are determined using Kane's dynamical equations. Inertia, internal, control, and gravity forces are included to develop modes shapes of vibrations. Deflections at end points are then determined, consequently mass centre position along with beam orientation is amended to have precise positions of end point, where docking with other objects are attained.
\end{abstract}

Keywords: Elasticity of beams, Kane's dynamical equation, vibration, LEO maneuver.

\section{Introduction}

A growing concern has been rosin during the last decade in assembling large structures on orbit. These structures are ranging from mirrors to reflect the sun light on solar arrays [1], antennae [2,3,4], manipulators, [5], up to the ISS [6]. It is essentially to use light, consequently flexible, structure elements. Due to this flexibility, significant deflection and vibration assist. Accurate rendezvous and docking operations requires a complete knowledge about positions and velocities of connection points, normally end points [7].

Motion planning of a manoeuvring object usually considers the translation of mass centre and rotation about it. These manoeuvres are generated due to the requirements of reaching goal positions and orientation in the mean time avoiding obstacles which are in fact others manoeuvring objects. Goal configuration are determine through constructing the layout of the assembled structures based on all elements remain straight, which of course not the case [8].

A general dynamics of the beam are modelled by using Lagrangian approach. Position and velocity of end points relative to a body reference frame centred at the manoeuvring object mass centre is presented in this paper using Kanne's dynamical equations. Equations governing motions of the manoeuvring object is obtained by utilizing generalized speeds related to vibration modes $[9,10]$.

\section{Mode Shapes of Free-Vibration}

A free-flying elastic beam of length, $L$, constant flexural rigidity $E I$, and constant mass per unit length, $\rho$, is vibrated freely under the action of its own velocity and angular velocity. The governing partial differential equation for this case is defined as [11]:

\footnotetext{
*Egyptian Armed Forces, Egypt, ahmed.badawy@1ycos.com 


$$
E I \frac{\partial^{4} y}{\partial x^{4}}+\rho \frac{\partial^{2} y}{\partial t^{2}}=0
$$

The general solution has a form of

$$
y(x, t)=\sum_{i=1}^{\infty} W_{i}(x) T_{i}(t)
$$

The free-free boundary conditions are then defined as:

$$
y^{\prime \prime}(0, t)=y^{\prime \prime \prime}(0, t)=y^{\prime \prime}(L, t)=y^{\prime \prime \prime}(L, t)=0
$$

Normal functions for free-free vibration are:

$$
\begin{gathered}
W_{i}(x)=\sin \left(\frac{\lambda_{i} x}{L}\right)+\sinh \left(\frac{\lambda_{i} x}{L}\right)+\frac{\sin \left(\frac{\lambda_{i} x}{L}\right)-\sinh \left(\frac{\lambda_{i} x}{L}\right)}{\cosh \left(\frac{\lambda_{i} x}{L}\right)-\cos \left(\frac{\lambda_{i} x}{L}\right)}\left(\cos \left(\frac{\lambda_{i} x}{L}\right)+\cosh \left(\frac{\lambda_{i} x}{L}\right)\right) \\
T_{i}(t)=\alpha_{i} \cos \left(p_{i} t\right)+\beta_{i} \sin \left(p_{i} t\right)
\end{gathered}
$$

where $\lambda_{i}$ are the roots of the following transcendental equation:

$$
\cos \lambda \cosh \lambda-1=0
$$

and

$$
p_{i}=\left(\frac{\lambda_{i}}{L}\right)^{2} \sqrt{\frac{E I}{\rho}}
$$

Whereas $\alpha_{i}$ and $\beta_{i}$ are constants depend on initial conditions. Normal functions satisfy the orthogonality condition.

$$
\int_{0}^{L} W_{i} W_{j} \rho d x=m \delta_{i j}, \quad(i, j=1,, 2, \ldots, \infty)
$$

and

$$
E I \int_{0}^{L} W_{i}^{\prime} W_{j}^{\prime \prime} d x=p_{i}^{2} m \delta_{i j}, \quad(i, j=1,, 2, \ldots, \infty)
$$

where

$$
\begin{aligned}
& \mathrm{m} \ldots \text { beam mass } \\
& \delta_{i j} \ldots \text { Kronecker delta }
\end{aligned}
$$




\section{Motion in Earth Orbit}

The maneuvering beam is placed in earth gravitational field. Earth is considered as a fixed particle in an inertial frame of reference and is designated as particle $E$ of mass $m_{\mathrm{E}}$. Two external systems of forces are considered: gravitational force due to the existence of particle $\mathrm{E}$ and control actions, Fig. 1.

Centre of gravity of the beam, point $A$, is characterized by three generalized speeds in the inertial frame of reference $u_{1}, u_{2}$ and $u_{3}$ where [10]:

$$
\begin{aligned}
& u_{1}={ }^{N} \mathbf{v}_{A} \cdot \mathbf{a}_{1} \\
& u_{2}={ }^{N} \mathbf{v}_{A} \cdot \mathbf{a}_{2} \\
& u_{3}={ }^{N} \boldsymbol{\omega . \mathbf { a } _ { 3 }}
\end{aligned}
$$

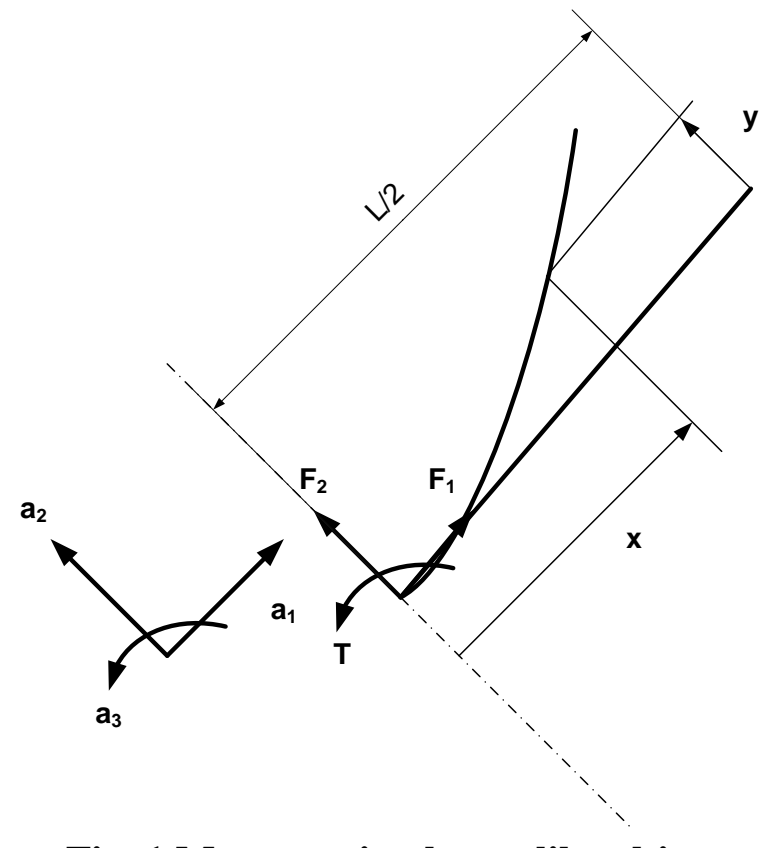

Fig. 1 Maneuvering beam-like object

where ${ }^{N} \mathbf{v}_{A}$ is the velocity of point $A$ in the inertial frame, $N$, and $\mathbf{a}_{\mathrm{i}}$ are unit vectors of frame $N$ and also fixed to point $A$, then

$$
\begin{aligned}
& { }^{N} \mathbf{v}_{A}=u_{1} \mathbf{a}_{1}+u_{2} \mathbf{a}_{2} \\
& { }^{N} \boldsymbol{\omega}=u_{3} \mathbf{a}_{3}
\end{aligned}
$$

Deformation of any point at distance $x$ from $A$, and generalized speeds are expressed considering $n$-normal functions as:

$$
\begin{gathered}
y(x, t)=\sum_{i=1}^{n} W_{i}(x) T_{i}(t) \\
u_{3+i}=\dot{T}_{i}(t) \quad i=1, \ldots, n
\end{gathered}
$$

Velocity of a generic point $P$ at distance $x$ from point $A$ is proved to be [10]: 


$$
{ }^{N} \mathbf{v}_{P}=\left(u_{1}-u_{3} \sum_{i=1}^{n} W_{i}(x) T_{i}(t)\right) \mathbf{a}_{1}+\left(u_{2}+x u_{3}+\sum_{i=1}^{n} W_{i}(x) u_{3+i}\right) \mathbf{a}_{2}
$$

The angular acceleration of the beam is:

$$
N_{\boldsymbol{\alpha}}=\dot{u}_{3} \mathbf{a}_{3}
$$

The acceleration of point $A$ is expressed as:

$$
\begin{aligned}
{ }^{N} \mathbf{a}_{A} & ={ }^{N} \dot{\mathbf{v}}_{A}{ }^{+}{ }^{N} \boldsymbol{\omega} \times{ }^{N} \mathbf{v}_{A} \\
& =\dot{u}_{1} \mathbf{a}_{1}+\dot{u}_{2} \mathbf{a}_{2}+u_{3} \mathbf{a}_{3} \times\left(u_{1} \mathbf{a}_{1}+u_{2} \mathbf{a}_{2}\right) \\
& =\left(\dot{u}_{1}-u_{3} u_{2}\right) \mathbf{a}_{1}+\left(\dot{u}_{2}+u_{3} u_{1}\right) \mathbf{a}_{2}
\end{aligned}
$$

Acceleration of a generic point $P$ at distance $x$ from point $A$ is proved to be:

$$
\begin{aligned}
& { }^{N} \mathbf{a}_{P}={ }^{N} \mathbf{a}_{A}{ }^{+}{ }^{N} \mathbf{a}_{P / A}{ }^{+}{ }^{N} \mathbf{a}_{P, c o r} \\
& =\left(\dot{u}_{1}-u_{3} u_{2}\right) \mathbf{a}_{1}+\left(\dot{u}_{2}+u_{3} u_{1}\right) \mathbf{a}_{2}-\left(x u_{3}^{2} \mathbf{a}_{1}+u_{3}^{2} \sum_{i=1}^{n} W_{i}(x) T_{i}(t) \mathbf{a}_{2}\right)+ \\
& \left(-\dot{u}_{3} \sum_{i=1}^{n} W_{i}(x) T_{i}(t) \mathbf{a}_{1}+x \dot{u}_{3} \mathbf{a}_{2}\right)+2 u_{3}\left(\sum_{i=1}^{n} W_{i}(x) u_{3+i}\right) \mathbf{a}_{1}+\sum_{i=1}^{n} W_{i}(x) \dot{u}_{3+i} \mathbf{a}_{2} \\
& { }^{N} \mathbf{a}_{P}=\left(\dot{u}_{1}-u_{3} u_{2}-x u_{3}^{2}-\sum_{i=1}^{n} W_{i}(x)\left(\dot{u}_{3} T_{i}(t)+2 u_{3} u_{3+i}\right)\right) \mathbf{a}_{1} \\
& +\left(\dot{u}_{2}+u_{3} u_{1}+x \dot{u}_{3}+\sum_{i=1}^{n} W_{i}(x)\left(\dot{u}_{3+i}-u_{3}^{2} T_{i}(t)\right)\right) \mathbf{a}_{2}
\end{aligned}
$$

\section{Generalized Inertia Force}

Total generalized inertia force for a beam of length, $L$, is defined as:

$$
\mathbf{F}_{j}^{I}=-\int_{0}^{L} \frac{\partial^{N} \mathbf{v}_{P}}{\partial u_{j}} \cdot{ }^{N} \mathbf{a}_{P} \rho d x \quad, j=1, \ldots, 3+n
$$

Substituting from eqs. $(14,18)$ in the previous equation gives: 


$$
\begin{aligned}
\mathbf{F}_{1}^{I}= & -\int_{0}^{L}\left[\dot{u}_{1}-u_{3} u_{2}-x u_{3}^{2}-\sum_{i=1}^{n} W_{i}(x)\left(\dot{u}_{3} T_{i}(t)+2 u_{3} u_{3+i}\right)\right] \rho d x \\
& =-m\left(\dot{u}_{1}-u_{3} u_{2}\right)+u_{3}^{2} \int_{0}^{L} x \rho d x+\dot{u}_{3} \sum_{i=1}^{n}\left(\int_{0}^{L} W_{i}(x) \rho d x\right) T_{i}(t)+2 u_{3} \sum_{i=1}^{n} \int_{0}^{L}\left(W_{i}(x) \rho d x\right) u_{3+i} \\
& \mathbf{F}_{2}^{I}=-m\left(\dot{u}_{2}+u_{3} u_{1}\right)-\dot{u}_{3} \int_{0}^{L} x \rho d x-\sum_{i=1}^{n}\left(\int_{0}^{L} W_{i}(x) \rho d x\right) \dot{u}_{3+i}+u_{3}^{2} \sum_{i=1}^{n} \int_{0}^{L}\left(W_{i}(x) \rho d x\right) T_{i}(t) \\
\mathbf{F}_{3}^{I}=-\left(\dot{u}_{2}+u_{3} u_{1}\right) \int_{0}^{L} x \rho d x+\left(\dot{u}_{1}-u_{2} u_{3}\right) \sum_{i=1}^{n}\left(\int_{0}^{L} W_{i}(x) \rho d x\right) T_{i}(t)- & \dot{u}_{3}^{L} x^{2} \rho d x-\sum_{i=1}^{n} \dot{u}_{3+i}\left(\int_{0}^{L} x W_{i}(x) \rho d x\right) \\
& \mathbf{F}_{3+j}^{I}=-\dot{u}_{2} \int_{0}^{L} W_{i}(x) \rho d x-\sum_{i=1}^{n}\left(\int_{0}^{L} W_{i}(x) W_{j}(x) \rho d x\right) \dot{u}_{3+i}-\dot{u}_{3} \int_{0}^{L} x W_{i}(x) \rho d x- \\
0 & u_{1} u_{3} \int_{0}^{L} W_{i}(x) \rho d x+u_{3}^{2} \sum_{i=1}^{n}\left(\int_{0}^{L} W_{i}(x) W_{j}(x) \rho d x\right)
\end{aligned}
$$

These generalized inertia forces are produced by internal, gravitational, and control forces.

\section{Internal Actions in the Beam}

Consider an element at distance, $x$, from point $A$, and of length $d x$, internal force is then described as:

$$
d \mathbf{F}=-\frac{\partial V}{\partial x} d x \mathbf{a}_{2}
$$

where $V(x)$ is the shear force at point $P$, Moreover neglecting the rotary inertia, the shear force is the derivative of bending moment at the point.

$$
V=\frac{\partial M}{\partial x}
$$

and

$$
M=E I \frac{\partial^{2} y}{\partial x^{2}}
$$

Then

$$
d \mathbf{F}=-\frac{\partial^{2}}{\partial x^{2}}\left(E I \frac{\partial^{2} y}{\partial x^{2}}\right) d x \mathbf{a}_{2}
$$


Total internal force is then defined as:

$$
\begin{aligned}
& \mathbf{F}_{j}^{i n}=\left|E I \frac{\partial^{N} \boldsymbol{\omega}}{\partial u_{j}} \cdot \mathbf{a}_{3} \frac{\partial^{2} y}{\partial x^{2}}-\frac{\partial^{N} \mathbf{v}}{\partial u_{j}} \cdot \mathbf{a}_{2} \frac{\partial^{3} y}{\partial x^{3}}\right|_{x=0}-\int_{0}^{L} \frac{\partial^{N} \mathbf{v}_{P}}{\partial u_{j}} \cdot \mathbf{a}_{2} \frac{\partial^{2}}{\partial x^{2}}\left(E I \frac{\partial^{2} y}{\partial x^{2}}\right) d x \\
& , j=1, \ldots, 3+n
\end{aligned}
$$

Apply boundary conditions as:

$$
W_{i}^{\prime \prime}(0)=W_{i}^{\prime \prime}(L)=W_{i}^{\prime \prime \prime}(0)=W_{i}^{\prime \prime \prime}(L)=0
$$

Then

$$
F_{1}^{i n}=F_{2}^{i n}=F_{3}^{i n}=0
$$

and

$$
F_{3+j}^{i n}=-\sum_{i=1}^{n}\left(\int_{0}^{L} E I W_{i}^{\prime \prime}(x) W_{j}^{\prime \prime}(x) d x\right) T_{i}(t) \quad, j=1, \ldots, n
$$

\section{Gravitational Force Exerts on the Beam}

Consider the sole attraction exerts the beam is due to the earth as a central body. The position vector of the centre of gravity, point $A$ in our case of homogeneous beam, is termed $R$. Position of any generic point $P$ from $P$ toward earth is then defined as:

$$
\mathbf{P}=R \mathbf{e}_{\mathbf{g}}-x \mathbf{a}_{1}-y \mathbf{a}_{2}
$$

Force on differential element at point $P$ is then:

$$
\begin{gathered}
d \mathbf{F}=\frac{G m_{E}(\rho d x)}{\|\mathbf{P}\|^{2}} \frac{\mathbf{P}}{\|\mathbf{P}\|} \\
d \mathbf{F}=\frac{G m_{E} \rho}{\|\mathbf{P}\|^{3}} \mathbf{P} d x
\end{gathered}
$$

Substituting and dropping high order terms in $\frac{x}{R}$ or $\frac{y}{R}$, then:

$$
\begin{array}{r}
d \mathbf{F}=\frac{G m_{E} \rho}{\|\mathbf{P}\|^{2}}\left[\left(-\cos (\theta)+\frac{3 x\left(\cos ^{2}(\theta)-1\right)-3 y \sin (\theta) \cos (\theta)}{R}\right) \mathbf{a}_{1}+\right. \\
\left.\left(\sin (\theta)-\frac{3 x \sin (\theta) \cos (\theta)+y\left(1-3 \sin ^{2}(\theta)\right)}{R}\right) \mathbf{a}_{2}\right]
\end{array}
$$

Total gravitational force is then expressed as:

$$
\mathbf{F}_{j}^{G}=\int_{0}^{L} \frac{\partial^{N} \mathbf{v}_{P}}{\partial u_{j}} \cdot d \mathbf{F} \quad, j=1, \ldots, 3+n
$$




\section{Control Actions}

Consider the maneuvering beam is controlled through couple moment at point $A$ and force at the same point in a general direction in the plane of motion. These actions are then expressed as:

$$
\mathbf{T}^{c}=T \mathbf{a}_{3}
$$

and

$$
\mathbf{F}^{c}=F_{1} \mathbf{a}_{1}+F_{2} \mathbf{a}_{2}
$$

Equivalent control force is expressed as:

$$
\mathbf{F}_{j}^{c}=\frac{\partial^{N} \boldsymbol{\omega}}{\partial u_{j}} \cdot \mathbf{T}^{c}+\frac{\partial^{N} \mathbf{v}_{A}}{\partial u_{j}} \cdot \mathbf{F}^{c} \quad, j=1, \ldots, 3+n
$$

\section{Kane's Dynamical Equations}

For a system in an inertial frame of reference, the summation of generalized active forces and generalized inertia forces are zero, then [9]:

$$
\mathbf{F}_{j}+\mathbf{F}_{j}^{I}=0 \quad, j=1, \ldots, 3+n
$$

Or

$$
\mathbf{F}_{j}^{i n}+\mathbf{F}_{j}^{G}+\mathbf{F}_{j}^{c}+\mathbf{F}_{j}^{I}=0 \quad, j=1, \ldots, 3+n
$$

Using equations (20), (25), (30) and (33) along with eq.(35) give:

$$
\begin{aligned}
& m\left(\dot{u}_{1}-u_{3} u_{2}\right)-\frac{\rho L^{2}}{2} u_{3}^{2}-\dot{u}_{3} \sum_{i=1}^{n}\left(\int_{0}^{L} W_{i}(x) \rho d x\right) T_{i}(t)-2 u_{3} \sum_{i=1}^{n} \int_{0}^{L}\left(W_{i}(x) \rho d x\right) u_{3+i}+ \\
& \frac{G m_{E}}{R^{2}}\left[\left(m \cos (\theta)+\frac{\left(1-3 \cos ^{2}(\theta)\right) \rho L^{2}}{2 R}+\frac{3 \sin (\theta) \cos (\theta)}{R} \sum_{i=1}^{n}\left(\int_{0}^{L} W_{i}(x) \rho d x\right) T_{i}(t)\right)-F_{1}=0\right. \\
& m\left(\dot{u}_{2}+u_{3} u_{1}\right)+\frac{\rho L^{2}}{2} \dot{u}_{3}+\sum_{i=0}^{n}\left(\int_{0}^{L} W_{i}(x) \rho d x\right) \dot{u}_{3+i}-u_{3}^{2} \sum_{i=1}^{n} \int_{0}^{L}\left(W_{i}(x) \rho d x\right) T_{i}(t)+ \\
& \frac{G m_{E}}{R^{2}}\left[\left(-m \sin (\theta)+\frac{3 \sin (\theta) \cos (\theta)}{2 R} \rho L^{2}+\frac{1-3 \sin ^{2}(\theta)}{R} \sum_{i=1}^{n}\left(\int_{0}^{L} W_{i}(x) \rho d x\right) T_{i}(t)\right)-F_{2}=0\right.
\end{aligned}
$$




$$
\begin{aligned}
& -\frac{\rho L^{2}}{2}\left(\dot{u}_{2}+u_{3} u_{1}\right)+\left(\dot{u}_{1}-u_{2} u_{3}\right) \sum_{i=0}^{n}\left(\int_{0}^{L} W_{i}(x) \rho d x\right) T_{i}(t)-\dot{u}_{3} \frac{\rho L^{3}}{3}-\sum_{i=1}^{n} \dot{u}_{3+i}\left(\int_{0}^{L} x W_{i}(x) \rho d x\right)+ \\
& \frac{G m_{E}}{R^{2}}\left[\frac{\rho L^{2}}{2} \sin (\theta)-\frac{\rho L^{3} \sin (\theta) \cos (\theta)}{R}+\right. \\
& \left.\frac{1}{R} \sum_{i=1}^{n}\left(\left(\int_{0}^{L} W_{i}(x) \rho d x\right) \cos (\theta)+3\left(\int_{0}^{L} x W_{i}(x) \rho d x\right)\left(1-2 \cos ^{2}(\theta)\right)\right) T_{i}(t)\right]+T=0 \\
& \left(\dot{u}_{2}+u_{1} u_{3}\right) \int_{0}^{L} W_{i}(x) \rho d x+\sum_{i=1}^{n}\left(\int_{0}^{L} W_{i}(x) W_{j}(x) \rho d x\right) \dot{u}_{3+i}+\dot{u}_{3} \int_{0}^{L} x W_{i}(x) \rho d x+ \\
& +\sum_{i=1}^{n}\left(\int_{0}^{L} E I W_{i}^{\prime \prime}(x) W_{j}^{\prime \prime}(x) d x\right) T_{i}(t)-\mathrm{u}_{3}^{2} \sum_{i=1}^{n}\left(\int_{0}^{L} W_{i}(x) W_{j}(x) \rho d x\right) T_{i}(t)+ \\
& \frac{G m_{E}}{R^{2}}\left[-\sin (\theta) \int_{0}^{L} W_{i}(x) \rho d x+\frac{3 \sin (\theta) \cos (\theta)}{R} \int_{0}^{L} x W_{i}(x) \rho d x\right. \\
& \left.+\frac{\left(1-3 \sin ^{2}(\theta)\right)}{R} \sum_{i=1}^{n}\left(\int_{0}^{L} W_{i}(x) W_{j}(x) \rho d x\right) T_{i}(t)\right]=0 \quad j=1, \ldots, n
\end{aligned}
$$

\section{Analysis of Kane's Equations}

Kane's dynamical equations give $(3+n)$ equations in the following unknowns $\left(\mathrm{T}_{1}, \ldots, \mathrm{T}_{\mathrm{n}}, \mathrm{u}_{1}, \ldots, \mathrm{u}_{3+\mathrm{n}}\right)$. Additional kinematical equations are needed to solve this system of equations. These equations are provided as:

$$
u_{3+i}=\dot{T}_{i} \quad, i=1, \ldots, n
$$

Using eq. (4) and performing integrations throughout eqs. (36-39) lead to the following:

$$
\begin{gathered}
\int_{0}^{L} W_{i}(x) \rho d x=\frac{2 m\left(1+e^{-2 \lambda_{i}}+2 e^{-\lambda_{i}} \cos \lambda_{i}\right)}{\lambda_{i}\left(1-e^{-2 \lambda_{i}}+2 e^{-\lambda_{i}} \sin \lambda_{i}\right)} \\
\int_{0}^{L} x W_{i}(x) \rho d x=\frac{2 m L}{\lambda_{i}^{2}} \\
\int_{0}^{L} W_{i}(x) W_{j}(x) \rho d x=m \delta_{i j} \\
\int_{0}^{L} E I W_{i}^{\prime \prime}(x) W_{j}^{\prime \prime}(x) d x=\frac{\lambda_{i}^{4} E I}{L^{3}}
\end{gathered}
$$




\section{Numerical Results}

For a maneuvering elastic beam like structure, the control algorithm provides us with controller interventions as impulses. These impulsive forces are only applied when the natural motion of the maneuvering elements does not provide the required rate of approaching the goal configuration and avoiding obstacles, Fig. 2.

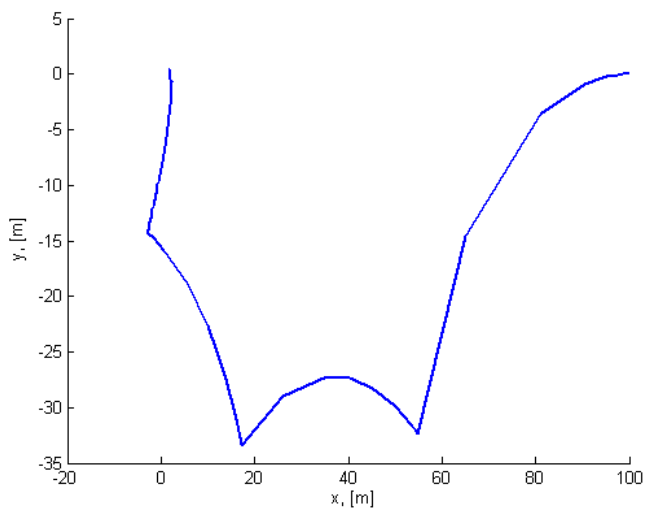

Object maneuver in plane

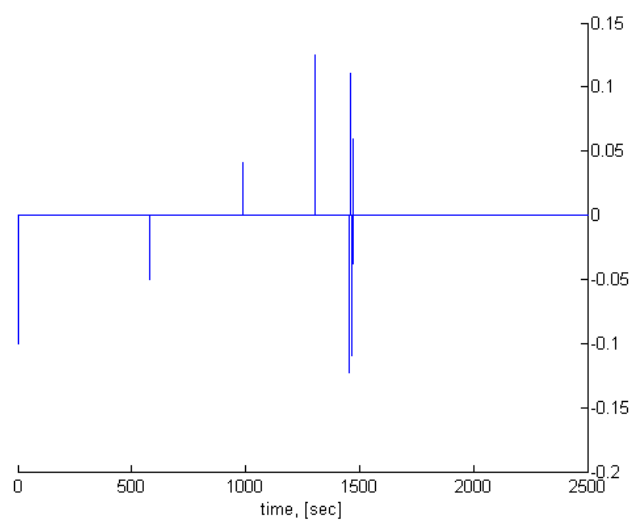

Impulsive force

Fig. 2 Control demand (courtesy of [8])

Impulsive forces produced from control algorithm is now provided to the dynamic model to calculate the deflection and velocity of end points for the manoeuvring beam like object with the data given in Table 1.

Table. 1 Object properties

\begin{tabular}{|c|c|c|c|}
\hline length $[\mathrm{m}]$ & Density, $[\mathrm{kg} / \mathrm{m}]$ & Altitude, $[\mathrm{km}]$ & Flexural rigidity, $\left[\mathrm{N} \mathrm{m}^{2}\right]$ \\
\hline 20 & 0.2 & 1000 & 5 \\
\hline
\end{tabular}

Results for beam deflections are presented in fig. 3. These results are provided to the controller to amend its goal position to make these variations in end position with docking mechanism tolerance. 

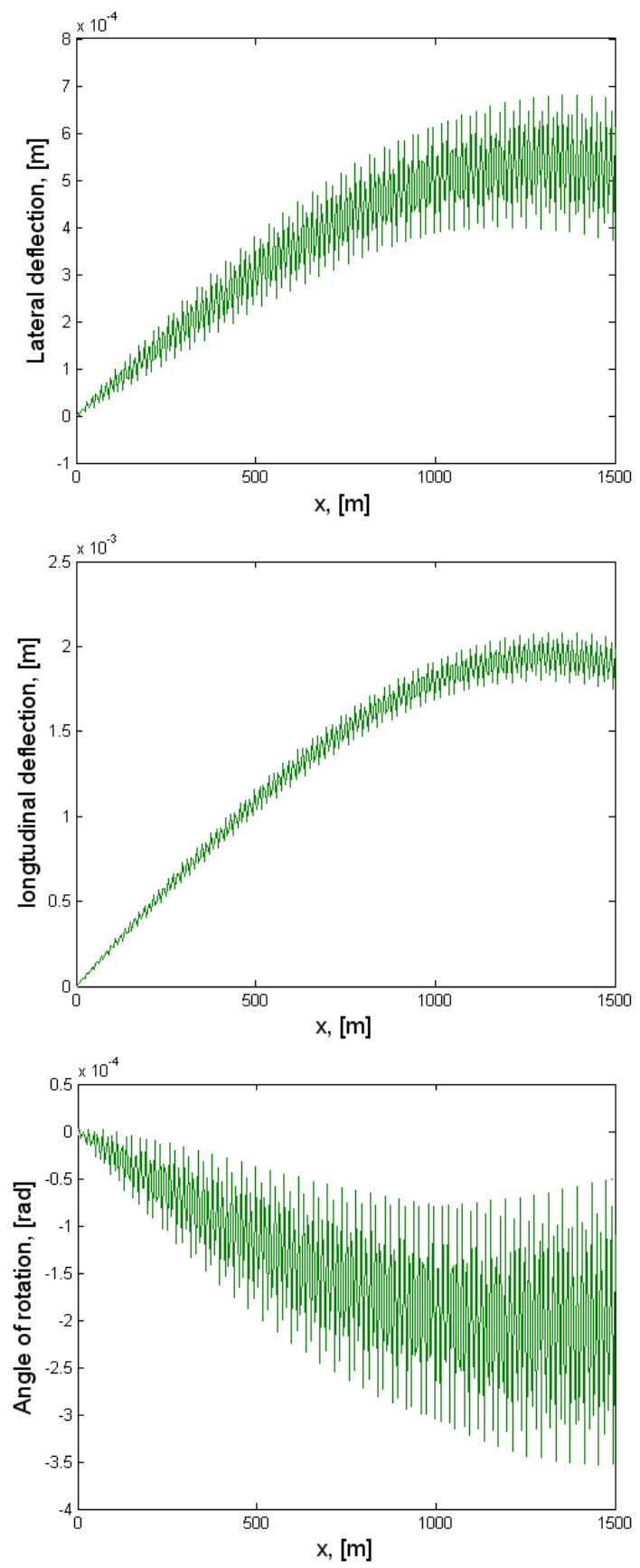

Fig. 3 End point Deflection and Rotation

Mass centre velocity and angular velocity are also provided to the controller to estimate the actual position and orientation to be forwarded to the next phase on control action when demanded. 

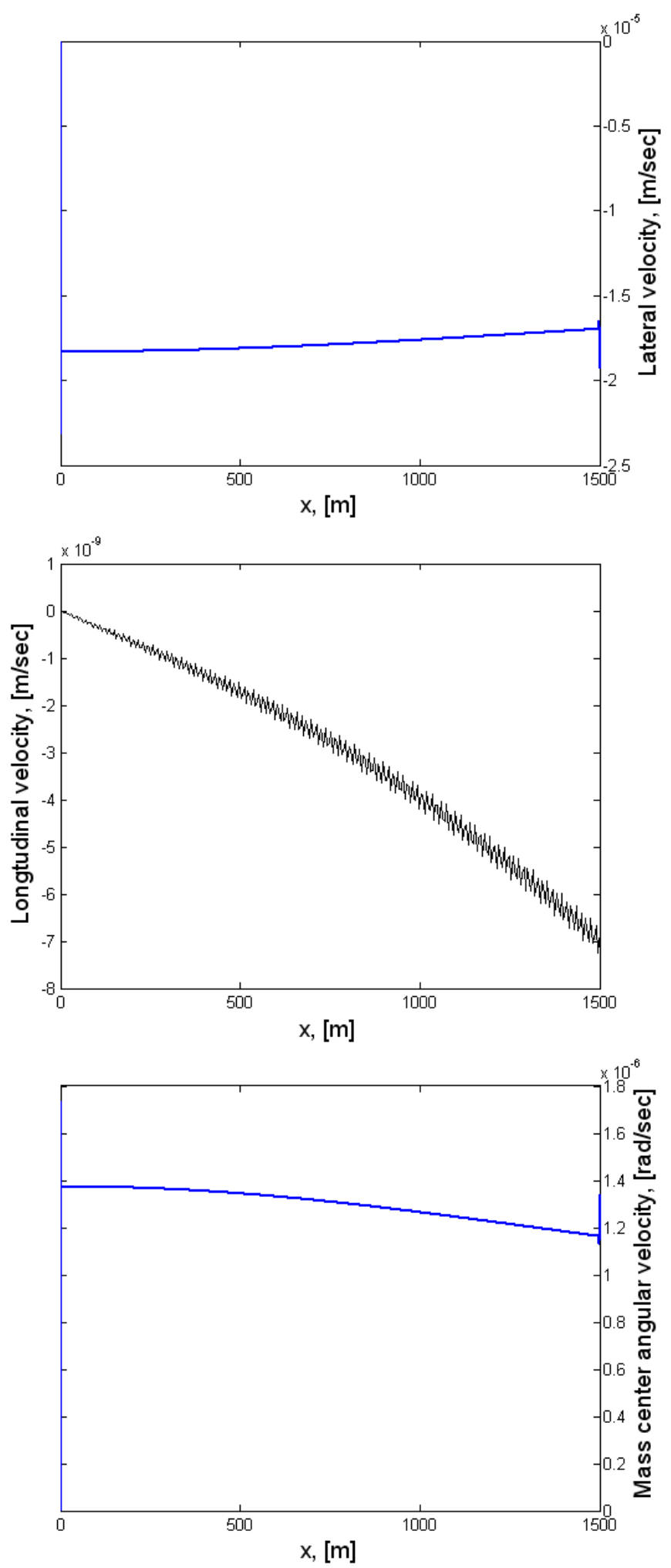

Fig. 4 Mass Centre Velocity and Angular Velocity 


\section{Conclusions}

Dynamic model along with control algorithm prove their ability to perform successful onorbit mission for flexible beam-like structure. Determination of the required controller intervention for a rigid body is the first step in mission accomplishment, which is considered herein as a preliminary estimation for the required force and velocity. The dynamic model is then thriving efficiently in estimating the consequences of applying such control forces on both end and mass centre points. These data are now sent back to the controller to adjust thruster performance according to the actual flexible maneuvering object status.

\section{References}

[1] Vasile M., et. al., "Mirror Formation Control in the Vicinity of an Asteroid", AIAA/AAS Astrodynamics Specialist Conference and Exhibit, Honolulu, Hawaii, AIAA 2008- 6252.

[2] $\mathrm{Xu} \mathrm{Y}$. and Guan F., "Structure Design and Mechanical Measurement of Inflatable Antenna", Acta Astronautica, 76 (2012) 13-25.

[3] Zhang Y. et. al., "A Controlled Deployment Method for Flexible Deployable Space Antennas", Acta Astronautica 81 (2012) 19-29.

[4] Li T. and Wang Y., "Performance Relationships between Ground Model and Space Prototype of Deployable Space Antennas", Acta Astronautica 65 (2009) 1383-1392.

[5] Book W. J., "Recursive Lagrangian Dynamics of Flexible Manipulator Arms via Transformation Matrices", Carncgic- Mellon University, 1983.

[6] Badawy A., and McInnes C.R., "Free Flyer Maneuvering round a Space Station", The Twelfth International Conference on Aerospace Science and Aviation Technology, ASAT-12, Cairo, Egypt, 2007, paper no. 36.

[7] Zhang X., et. al., "Research of Flexible Beam Impact Dynamics based on Space Probecone Docking Mechanism”, Advances in Space Research 49 (2012) 1053-1061.

[8] Badawy A., "On-orbit Maneuvering using Superquadric Potential Fields", PhD thesis, University of Strathclyde, UK, 2007.

[9] Kane T R. and Levinson D. A., "Dynamics Theory and Applications", McGraw-Hill Book Company, 1985.

[10] Kane T. R.. et. al., "Spacecraft Dynamics”, McGraw-Hill Book Company, 1993.

[11] Rao S. S., "Mechanical vibration", Addison-Wesley Publishing Company, 2000. 